Ma. Victoria P. Pascual, MD

Daniel M. Alonzo, MD

Department of Otorhinolaryngology

Head and Neck Surgery

The Medical City, Ortigas City
Correspondence: Dr. Daniel M. Alonzo

Department of Otorhinolaryngology-Head and Neck Surgery

The Medical City

Ortigas Avenue, Pasig City 1600

Philippines

Phone: (632) 6356789 local 6250

Fax: (632) 6873349

Email:ent@themedicalcity.com

The authors declare that this represents original material that is not being considered for publication elsewhere in full or in part, in print or electronic media; that the manuscript has been read and approved by all the authors, that the requirements for authorship have been met by each authors, and that each author believes that the manuscript represents honest work.

Disclosures: The authors signed disclosures that there are no financial or other (including personal) relationships, intellectual passion, political or religious beliefs, and institutional affiliations that might lead to a conflict of interest

Presented at the Philippine Society of Otolaryngology-Head and Neck Surgery Clinical Case Report Contest (3rd Place), Menarini Office, 4/F W Bldg. 11th Ave. cor. 28th St. Bonifacio High St. BGC Taguig, June 2, 2015

\title{
Recurrent Epistaxis from a Post-Traumatic Infraorbital Artery Pseudoaneurysm
}

\begin{abstract}
Objective: To present a rare case of post-traumatic recurrent epistaxis in an elderly woman.
\end{abstract}

\section{Methods:}

Design: Case Report

Setting: Tertiary Private Hospital

Patient: One

Result: A 93-year-old woman had multiple admissions for recurrent life threatening nosebleeding that was not controlled until a post-traumatic pseudoaneurysm of the infraorbital artery was diagnosed and embolized.

Conclusion: The diagnosis of pseudoaneurysm should be considered in such cases, and treatment involving surgeons and interventional radiologists should be initiated to minimize morbidity and mortality.

Keywords: recurrent epistaxis, post-traumatic pseudoaneurysm, right infraorbital artery, right internal maxillary artery, embolization

Epistaxis is a common medical condition, with about $60 \%$ of the adult population having at least one episode during their lifetime.' Eighty to ninety percent are idiopathic, with about $80 \%$ occurring from the highly vascularized anterior nasal septum. ${ }^{2}$ Bleeding from this area is usually easily managed conservatively. However, when epistaxis is massive and intractable, differentials for possible sources include bleeding originating from the ethmoidal and sphenopalatine artery, facial and skull base trauma and post-traumatic aneurysms. ${ }^{3}$ We present an even rarer cause of such epistaxis. 


\section{CASE REPORT}

A 93-year-old woman consulted for bilateral epistaxis after falling and hitting her face and left shoulder on the ground. On examination, only blood clots in both nasal cavities and swelling and bruising of the right zygomatic area were noted. A facial CT scan showed a comminuted, depressed fracture of the right orbital floor, and hemoantrum of bilateral maxillary and right sphenoid sinus. The Ear Nose Throat - Head and Neck Surgery, Ophthalmology, and Emergency Room (ER) services determined that no immediate intervention was warranted, and discharged her with a nasal spray and antibiotics, advising outpatient follow-up.

Later that afternoon, she had right sided epistaxis amounting to approximately 2 cups while straining during defecation. The bleeding eventually stopped after cold compress, ice chips by mouth, and digital pressure. She was nevertheless admitted for observation and discharged a few days later with no recurrence of epistaxis during her hospital stay.

One week later, she was seen at the ER for right maxillary pain and numbness over the right nostril and upper lip. There had also been intermittent epistaxis at home of about $1 / 2$ cup per episode but spontaneously relieved each time. She was reevaluated and again sent home with an oral decongestant. But that evening, she was readmitted for post-tussive epistaxis amounting to 2 cups of blood.

During this admission, the patient was noted to have decreasing hemoglobin levels, attributed to intermittent episodes of right sided epistaxis of about $10 \mathrm{~mL}$ per episode, relieved spontaneously. After dismissing a hematologic etiology, a PNS CT scan revealed a comminuted, depressed fracture of the right orbital floor, hemoantrum within both maxillary and right ethmoid sinuses, and an ill-defined soft tissue density in the right middle meatus. (Figure 1) She was then scheduled for surgical exploration.

Intraoperative endoscopy revealed a hematoma in the maxillary ostium. Uncinectomy was performed for better visualization but was abandoned due to persistent bleeding. The approach was converted to a Caldwell incision, but profuse bleeding amounting to $700 \mathrm{~mL}$ within 2 minutes was immediately encountered upon entry into the maxillary sinus. Hemostasis was achieved with application of gauze impregnated with petroleum jelly into the sinus, and insertion of polyvinyl alcohol sponge (Merocel') into the anterior nasal cavity. She was then referred to Interventional Radiology, and diagnostic angiography was carried out the following day in the hopes of locating the bleeding vessel. The procedure revealed a pseudoaneurysm (PA) of the inferior orbital branch of the right internal maxillary artery (Figure 2), which was subsequently embolized using polyvinyl alcohol (PVA). Follow-up arteriography (Figure 3) still showed flow into the PA, and repeat embolization (Figure 4) was performed until the PA was occluded. The patient tolerated the procedure well with no noted complications or recurrence of epistaxis. The maxillary sinus and nasal packs were removed the next morning, and she was subsequently discharged 2 days later asymptomatic.

On follow-up one week from discharge, she reported complete resolution of epistaxis. She has better appetite and decreased right maxillary tenderness.
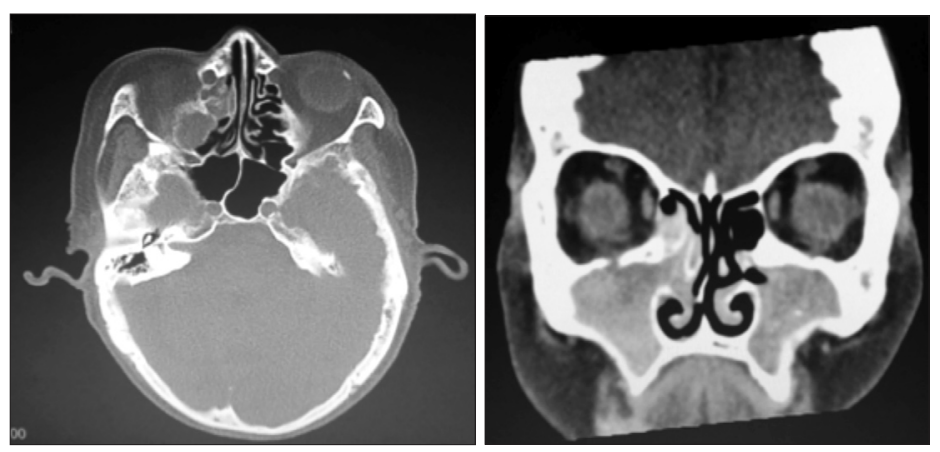

Figure 1. PNS CT scan with contrast (3 weeks post trauma). Comminuted, depressed fracture of the right orbital floor, hemoantrum of both maxillary and right ethmoid sinuses, and obstructed ostiomeatal units and right nasofrontal recess.

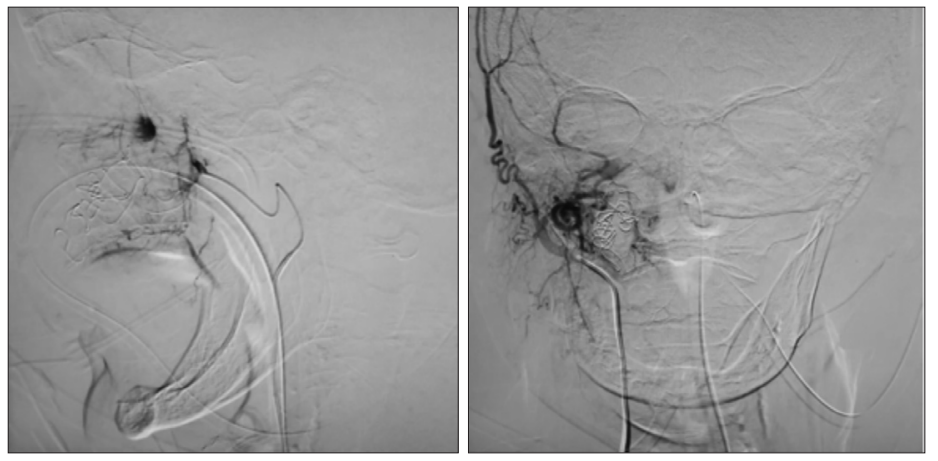

Figure 2. CT angiography. Left- sagittal view. Right- coronal view. Catheter inserted into the right common femoral artery and advanced into the right external carotid artery. Superselective arteriography performed, identifying a pseudoaneurysm from the inferior orbital artery branch of the right internal maxillary artery. Bleeding vessel symbolized by the extravasation of the dye. Initial embolization done using PVA.
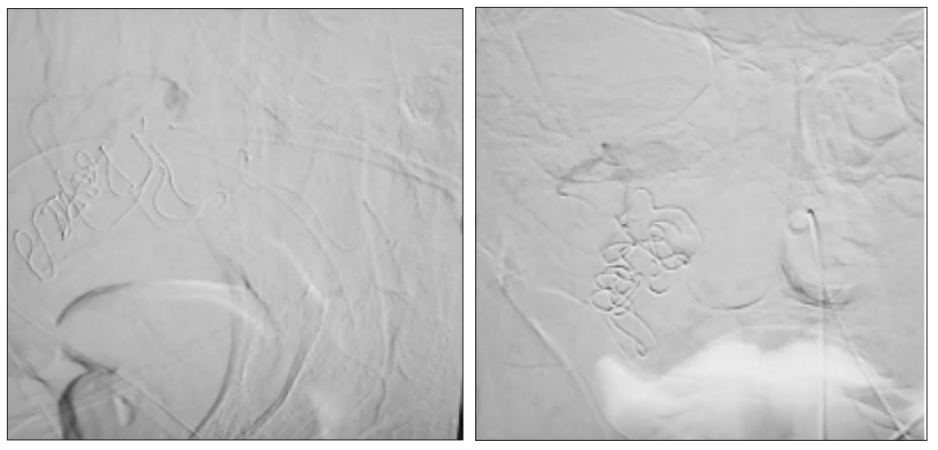

Figure 3. Arteriography after initial embolization. Note slower flow into the pseudoaneurysm but still with filling. Further selective arteriography performed until occlusion of the pseudoaneurysm was achieved. 

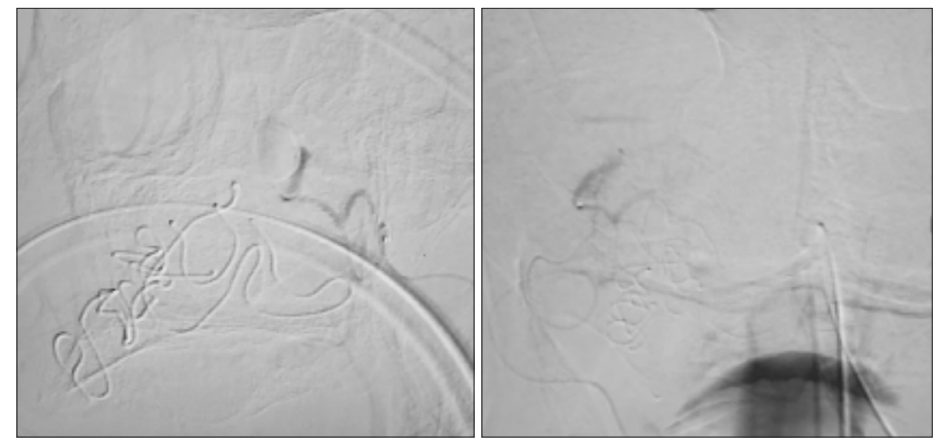

Figure 4. Repeat embolization. After the second attempt, no spillage of dye noted. No residual contrast media retention.

\section{DISCUSSION}

Pseudoaneurysm (PA) or false aneurysm is an outpouching of a blood vessel that results from disruption of one or more layers of its wall, rather than expansion of all wall layers as seen in a true aneurysm. ${ }^{4}$ Asymmetries, neurological deficits, and thromboembolism are the main signs and symptoms. ${ }^{4,5}$

Pseudoaneurysms can arise from the branches of the external carotid artery (ECA) or the internal carotid artery (ICA) system. ${ }^{5}$ Pseudoaneurysms of the ECA are rare. In a series of over 8,000 aneurysms, McCollum et al. reported only 21 PAs of the ECA, and 19 of them occurred after carotid surgery. ${ }^{6}$ This places its incidence at about $0.02 \%$. Its rarity might be attributed to the small size of the branches of the ECA. Due to its superficial course and its position as it crosses bony structures, the superficial temporal, distal facial and distal internal maxillary arteries are the most commonly involved vessels. ${ }^{3}$

The internal maxillary artery (IMA) is the largest terminal branch of the ECA, and bleeding from this site is mainly caused by bone fractures ${ }^{5}$ from direct penetrating or blunt trauma often fracturing the maxilla. ${ }^{6}$ Other possible causes include post-irradiation vasculopathy, tumor invasion in patients with head and neck cancers who received composite treatment, or maxillomandibular surgery. ${ }^{6,7}$

The hallmark of post-traumatic pseudoaneurysm (PTSA) is delayed, progressively more severe and sometimes fatal bleeding.' It can occur as early as four hours after the injury and as late as eight hours. Angiography is the most confirmatory test in diagnosis and is considered the gold standard investigation. ${ }^{5,6}$ It is characterized by residual contrast media retention in the lesion long after the arterial phase of diagnostic angiogram is done. ${ }^{6}$

Studies have shown that as many as $89 \%$ of untreated PA resolve in 5 to 90 days. ${ }^{3}$ However, in those with profuse life-threatening oronasal bleeding, initial management includes application of tight nasal and oral packing, followed by blood transfusion to maintain hemodynamic stability. More definitive treatments include emergent open surgical ligation of the IMA ${ }^{6}$ via arterial ligation methods, transantral approach to the maxillary artery, or external carotid artery ligation. However, these have high failure rates due to extensive anastomosis distal to the site of ligation. ${ }^{2}$

Current management of epistaxis includes angiography and embolization of the bleeding vessels. ${ }^{2}$ Endovascular embolization is the most successful way to manage PA of the IMA with rapid relief of symptoms. It causes reduction in blood flow, retrograde filling of the proximally occluded vessel and eventual obliteration of the PA. ${ }^{1}$ It has gained considerable clinical recognition because of its capability for super-selective localization of the bleeding, allowing preservation of all the other branches of the ECA. ${ }^{6}$ It allows more distal access to bleeding points, is less invasive, requires a shorter time for the procedure, and is faster than open arterial ligation. ${ }^{2}$

The common permanent embolic agents employed for endovascular embolization of vascular lesions in the head and neck region are polyvinyl particle (PVA) foam, microcoils, and liquid adhesives., ${ }^{1,3}$ PVA has been widely used for the treatment of idiopathic epistaxis and for preoperative tumor embolization' since it is the easiest to handle and has a higher capacity for vascular occlusion compared to metal coils. ${ }^{3}$ However, there is the risk of developing recurrent PAs with bleeding after embolization. ${ }^{1}$ Other complications range from minor transient adverse events like headache, facial pain or fever, to persistent complications such as monocular blindness, peripheral facial nerve paralysis or cerebral infarction. ${ }^{2}$

Epistaxis is an expected sequelae of trauma. However, persistent or recurrent bleeding and excessive blood loss are not usual. PTPA is rare but should raise a high index of suspicion when a patient presents with intractable epistaxis. 'Especially with geriatric patients whose clinical status may quickly deteriorate ${ }^{4}$ even with subtle changes from normal bodily functions, early diagnosis and treatment of a PA is imperative to minimize morbidity and mortality. As seen in our case, recurrent epistaxis should not be taken lightly.

\section{REFERENCES}

1. Willems PWA, Farb RI, Agid R. Endovascular Treatment of Epistaxis. AJNR Am J Neuroradiol. 2009 Oct: 30(9):1637-1645.

2. Haroon Y, Saleh HA, Al-Azzazy MZ, Abou-Issa AH. Embolization for control of refractory posterior epistaxis. EJRNM. 2012: 43(3):407-411.

3. Masooni A, Fazlipour A, Davoodi M. Traumatic Pseudoaneurysm of Internal Maxillary Artery: A Case Report. Vascular \& Interventional. Iran J Radiol. 2009:6(1):37-39.

4. Barbalho JCM, Santos ES, Menezes JMS Jr, Goncalves FR, Chagas OL Jr. Treatment of pseudoaneurysm of internal maxillary artery: a case report. Craniomaxillofac Trauma Reconstruc. 2010 Jun: 3(2):87-89.

5. Zhang CW, Xie XD, You C, Mao BY, Wang CH, He M, Sun H. Endovascular treatment of traumatic pseudoaneurysm presenting as intractable epistaxis. Korean J Radiol. 2010 Nov-Dec: 11(6):603 611.

6. Luo CB, Teng MM, Chang FC, Chang CY. Role of $\mathrm{CT}$ and endovascular embolization in managing pseudoaneurysms of the internal maxillary artery. J Chin Med Assoc. 2006 Jul; 69(7):310-16.

7. Karanth SK, Jagannathan M, Mahesh SG, Devale M. Internal maxillary artery pseudoaneurysm in a case of mandibular fracture. Indian J Plast Surg 2007:40(1):51-53. 